\title{
Location of Mobile Terminals Using Time Measurements and Survey Points
}

\author{
Michael McGuire, Member, IEEE, Konstantinos N. Plataniotis, Member, IEEE, and \\ Anastasios N. Venetsanopoulos, Fellow, IEEE
}

\begin{abstract}
Mobile terminal location has attracted much interest for its applications in emergency communications, location-sensitive browsing, and resource allocation. This paper introduces the use of nonparametric kernel-based estimators for location of mobile terminals using measurements of propagation delays. It is demonstrated that these estimators perform better than the previously used parametric maximum likelihood estimators for the case of a simulated microcell environment with line-of-sight (LOS) and non-line-of-sight (NLOS) radio propagation at several different levels of measurement noise. Their performance is not greatly degraded by NLOS effects. Methods for calculating good values for parameters of the kernel functions are demonstrated, as well as the robustness of the estimators when the values of the parameters vary from the optimal points. A lower bound on the mean square error of location estimation that considers the transition between LOS to NLOS propagation over short distances is presented. It is demonstrated the proposed location estimation method comes close to meeting this bound.
\end{abstract}

Index Terms-Land mobile radio cellular systems, position measurement, road-vehicle location monitoring.

\section{INTRODUCTION}

$\mathbf{T}$ HERE is much interest within the wireless communications research community on technologies that can estimate the location of mobile terminals. The reasons for location estimation technology can be classified into three categories: short-term, medium-term, and long-term.

The short-term reason for location estimation, in North America, is the Federal Communication Commission (FCC)'s (CRTC in Canada) requirement for mobile terminal location during emergency 911 (E-911) calls [1], [2]. The cellular provider is required to locate callers making E-911 calls to within $100 \mathrm{~m}$ over $67 \%$ of the time for network-based solutions. The cellular provider is expected to locate the callers making E-911 calls to within $50 \mathrm{~m}$ over $67 \%$ of the time for handset-based solutions. For handset-based solutions, the network providers have until December 2005 to have the location technology within $95 \%$ of all subscriber handsets in their network. The regulatory bodies in many other jurisdictions are considering similar regulations. Standardization of mobile terminal location technology is also taking place for GSM

Manuscript received November 2002; revised March 2003. The work of M. McGuire was supported in part by Nortel Institute for Telecommunications, University of Toronto, Toronto, ON, Canada.

The authors are with The Edward S. Rogers Sr. Department of Electrical and Computer Engineering, University of Toronto, Toronto, M5S 3G4 ON, Canada (e-mail: mmcguire@dsp.toronto.edu).

Digital Object Identifier 10.1109/TVT.2003.814222 networks [3]. The medium-term reasons for location-sensitive technologies are to create additional revenue opportunities for the network operators by allowing them to provide location-sensitive information browsing [4], vehicular fleet management, and intelligent traffic control [5]. The long-term reason for location technology is to assist resource allocation in the wireless network [6], [7]. It is desired that future wireless networks be able to provide multimedia communications, such as real-time video, that simultaneously require high bandwidth and high quality of service [8]. It has been shown that using location estimation and prediction for preemptive resource allocation is an attractive method of achieving this goal without requiring a daunting level of extra network capacity overhead.

Urban cells are the regions that produce the most revenue for network providers, so these are the regions of greatest interest for mobile terminal location algorithms. The users in these regions have the greatest demand for the wireless high-bandwidth multimedia services that are driving the development of next-generation wireless networks.

The importance of urban cells has created a demand for mobile terminal location algorithms that can perform well in this environment. For location of mobile terminals in urban areas, location algorithms based on propagation time measurements have been proposed [3], [9]. Urban cells have propagation conditions that can change from line-of-sight (LOS) to non-line-ofsight (NLOS) over a short distance [10]. The simplest propagation model is the LOS propagation model, where the shortest distance straight line path between the transmitter and receiver is unobstructed. NLOS propagation occurs when the LOS path is blocked by an obstacle and the radio signal travels via longer distance paths. Management of the two propagation types is a key issue of the mobile terminal location problem that has caused significant degradation of performance in the existing algorithms [11], [12].

The difficulty and importance of locating mobile terminals in urban areas has created much interest in this problem. To handle the measurement noise and changing propagation models, statistical estimation techniques are employed. If the joint probability density function for the measurement vector of propagation delay measurements and the location of the mobile terminal is known, then, given a measurement of delays for a mobile terminal, it is possible to calculate an optimal estimate of the mobile terminal's position [13].

In the urban cells that are discussed in this paper, the existence of multiple propagation conditions causes the joint probability density function to be complex and difficult to identify. The switching between NLOS and LOS propagation within small 
areas prevents parametric techniques from working well for estimating the joint probability density function. It would be necessary to identify and characterize the NLOS propagation regions. For this reason, a nonparametric technique where the joint probability density function is estimated as a sum of kernel functions based on a survey of propagation delays measured at known locations is proposed to solve this problem.

Section II contains a more detailed description of the measurements used to locate the mobile terminal. Different measurement types proposed to locate mobile terminals are listed, and the justification for the use of time measurements in this paper is provided. The models for the time measurements used by the estimators are outlined. Section III describes the nonparametric estimation technique. The probability density functions are approximated as a sum of kernel functions based on survey measurements, and numerical integration is performed to calculate the estimated positions. Rules for selecting the parameters for the estimation technique are presented. Section IV describes the configuration of Monte Carlo simulations of an urban environment with NLOS propagation used to evaluate the location estimation techniques. Section V contains the results of the simulation studies. The improvement that the nonparametric technique has over other techniques is shown. The performance of the new estimator is compared with the Weinstein-Weiss lower bound, which takes into account the environmental terrain and the prior probability density function of the mobile terminal location-factors not considered by previously presented lower bounds on location estimator performance. The lower bound allows estimator performance to be quantitatively appraised. Section VI summarizes our conclusions.

\section{MEASUREMENT MODEL}

Several methods have been proposed to locate wireless mobile terminals based on measuring the 1) angle of arrival (AoA) of the radio signal at base stations, 2) received signal strength (RSS), 3) time of arrival (ToA), and 4) time difference of arrival (TDoA) of the radio signals.

The AoA estimation technique requires that the base stations use specialized antennae to measure the bearing of incoming signals from the mobile terminals. If the bearing to the mobile terminal is known from two or more base stations, the location of the mobile terminal can be calculated. The biggest disadvantage of this technique is that the specialized antennas are expensive and not presently used for standard cellular networks [14]. This prevents AoA from being considered for any presently deployed networks.

The RSS technique has the advantage that it is fairly cheap since power measurements are already made by handsets as part of the handoff algorithm. If the propagation model is known, a power measurement can be mapped to a distance measurement. If the distance from the mobile terminal to three or more base stations is known, the mobile terminal position can be calculated. Unfortunately, RSS estimation error is higher than that of other localization methods. Also, the mobile terminal needs to be moving; otherwise, fast fading caused by multipath propagation causes disastrously high errors [15]. These factors prevent
RSS from being used as the sole means of locating mobile terminals, although its low cost does make it attractive for possible hybrid mobile terminal locating schemes.

The ToA and TDoA techniques are based on the measurement of propagation times. The code-division multiple-access (CDMA) receivers, proposed for most next-generation cellular systems, can make precise time measurements of the incoming signals using RAKE receivers [16]. A RAKE receiver estimates the delays of the propagation paths with the greatest signal powers. The estimated delay of the earliest detected propagation path for a base station is the measured propagation delay for that base station used for terminal location.

The ToA location technique involves measuring the time it takes a signal to travel from the mobile terminal to the base station. Making this measurement requires that the measuring device have accurate knowledge of the time of transmission [9]. This can be accomplished by synchronizing the transmitters and receivers, or by measuring the round-trip delay [3]. If the transmitters and receivers are synchronized and a RAKE receiver is used, highly accurate propagation time measurements can be made [9], [16]. Synchronizing the transmitters and receivers, the base stations and the mobile terminals in a cellular network, is costly. For round-trip measurements, a base station transmits a signal to the mobile terminal, which the mobile terminal immediately retransmits back to the base station. The round-trip delay is proportional to twice the radio propagation time plus processing delays. The resolution of this method is much less than that from using synchronized clocks but is much simpler to implement and less costly.

The TDoA location technique also involves measuring the propagation times but does not require that the measuring device know the times of transmission. The difference between the propagation times from the mobile terminal for pairs of base stations is measured. This can be accomplished two ways. If several base stations are synchronized, they can all transmit signals to the mobile terminal at the same time. Then the mobile can measure the difference between the propagation times for different base stations. Conversely, the mobile terminal can transmit a signal to the base stations. The base stations can measure the time they receive the mobile's signal and, by comparing their times, calculate the difference in propagation times [3]. If CDMA signals are used, the time measurements are accomplished using a RAKE receiver as for the ToA measurements. Only the base stations need to be synchronized. Synchronizing the base stations for TDoA measurements is much less expensive than synchronizing both the base stations and mobile terminals for ToA measurements [3].

Each time measurement defines for two-dimensional (2-D) location, in the case of LOS propagation and error-free measurements, a curve upon which the mobile terminal must reside. This curve is a circle for ToA measurements or a hyperbolic curve for TDoA measurements. Three ToA measurements or two TDoA measurements, both created from time measurements by three base stations, allow the mobile terminal position to be uniquely calculated [17].

Comparisons of location measurements in the literature have indicated that ToA/TDoA gives the lowest error [14], [15]. The use of ToA for the popular GPS geolocation systems proves that 
ToA can provide highly accurate location estimates. The channelization schemes proposed for next-generation cellular networks, such as CDMA and time-division multiple access, make the ToA and TDoA location schemes most attractive because the multiple-access schemes allow for high-accuracy time measurements [16], [18]. Because of these reasons, this paper concentrates on location based on ToA and TDoA measurements.

Complications in the mobile terminal location problem arise from two sources. First, the measurements in the field are not error-free, so that estimation techniques must take into account measurement noise. Second, the propagation in some cases is LOS and in others cases NLOS. In the NLOS case, the delay measurements will be biased from the LOS values and the simple geometric location techniques described above will lose accuracy or, in some cases, return ambiguous results. The simplest solution to these problems is to use more base stations' measurements. With more base stations, it is possible to average out the measurement noise, and base-station measurements with NLOS propagation can be detected and excluded from the estimation procedure [9], [12]. In real-world cellular networks, the number of base stations that can communicate with the mobile terminal is restricted, limiting the usefulness of excluding NLOS base stations [19]. Making extra measurements is also costly, so this paper concentrates on locating the mobile terminal with three base-station measurements, the minimum number required. The next section will provide an analysis of how the measuring base stations are selected.

Propagation time measurements are converted to propagation distance measurements by multiplication by $c$, the speed of light. We define $\mathbf{d}(\boldsymbol{\theta})$ as the vector of true propagation distances from the base stations to the mobile terminal when the mobile terminal is at location $\boldsymbol{\theta}$. This paper concentrates on 2-D location estimation since this is the case of greatest interest for wireless networks [5], [9]. The location vector is $\boldsymbol{\theta}=$ $(x, y)$, where $x$ is the $x$-coordinate and $y$ is the $y$-coordinate of the mobile terminal. The methods described here can be scaled up to three-dimensional location easily. If a simple one-corner diffraction model is used for NLOS propagation, as shown in Fig. 1, the $j$ th entry of $\mathbf{d}(\boldsymbol{\theta})$ is given by (1) at the bottom of the page, with $\left(x_{j}, y_{j}\right)$ being the location of the $j$ th base station and $\left(x_{c}, y_{c}\right)$ being the location of the corner. If the propagation is NLOS, the $j$ th entry of $\mathbf{d}(\boldsymbol{\theta})$ is a function not only of the mobile terminal and base stations' positions but also a function of the positions of the obstacles to the LOS propagation path. We will define the measured propagation distance vector $\tau$ as

$$
\boldsymbol{\tau}=\mathbf{d}(\boldsymbol{\theta})+\mathbf{v} .
$$

The $\mathbf{v}$ vector represents measurement noise with its $j$ th entry being the measurement noise for base station $j$. The dimensionality of the $\tau$ and $\mathbf{v}$ vectors is $k$, the number of base stations used to locate the mobile terminal. This paper discusses only

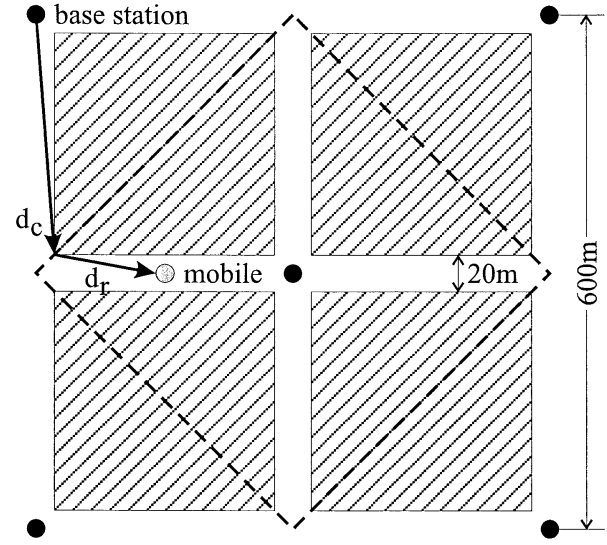

Fig. 1. Propagation environment.

the case when $k=3$, the minimum cost measurement case. The methods can be extended to greater $k$ with an expected improvement of performance as the estimation procedure is provided more information.

The measurement vector is denoted as $\mathbf{z}$ with a subscript indicating whether ToA or TDoA measurements are made. For ToA measurements, the measurement vector is defined as

$$
\mathbf{z}_{\mathrm{ToA}}=\boldsymbol{\tau} \text {. }
$$

Obviously, the length of the ToA measurement vector $\mathbf{z}_{\mathrm{ToA}}$ is $k$, the number of base-station measurements. For TDoA measurements, the measurement vector is defined as

$$
\mathbf{z}_{\mathrm{TDoA}}=\mathbf{D} \tau
$$

where $\mathbf{D}$ is a $(k-1 \times k)$ difference matrix of rank $(k-1)$. A difference matrix is mostly filled with zeros, with each row having a single entry of one and another entry of negative one. For example, if all TDoA measurements are made relative to the propagation time of base station one, the difference matrix is given by

$$
\mathbf{D}=\left[-1_{k-1} \vdots \mathbf{I}_{k-1}\right]
$$

where $\mathbf{1}_{k-1}$ is a length $(k-1)$ vector with all entries of one and $\mathbf{I}_{k-1}$ is a $(k-1 \times k-1)$ identity matrix. The TDoA measurement vector's length is $(k-1)$.

If it is assumed that the measurement noise for the propagation time for each base-station time measurement is independently and identically distributed, then the computation of the covariance of the measurement vectors $\mathbf{z}_{\mathrm{ToA}}$ and $\mathbf{z}_{\mathrm{TDoA}}$ given the mobile terminal location is trivial. The covariance of $\mathbf{z}$ and $\mathbf{C}$, using (3) and (4) for $k$ being the number of base-station measurements, is

$$
\mathbf{C}= \begin{cases}\sigma^{2} \mathbf{I}_{k} & \text { for ToA } \\ \sigma^{2} \mathbf{D D}^{T} & \text { for TDoA }\end{cases}
$$

$$
\mathbf{d}_{j}(\theta)= \begin{cases}\sqrt{\left(x-x_{j}\right)^{2}+\left(y-y_{j}\right)^{2}} & \text { LOS case } \\ \sqrt{\left(x-x_{c}\right)^{2}+\left(y-y_{c}\right)^{2}}+\sqrt{\left(x_{c}-x_{j}\right)^{2}+\left(y_{c}-y_{j}\right)^{2}} & \text { NLOS case }\end{cases}
$$


with $\sigma^{2}$ being the covariance of the propagation time measurement for a single base station (superscript $T$ denotes matrix transpose).

For general discussions of estimation technique applicable to either the ToA or TDoA methods or where the type of measurement data is clear from context, the subscript will be omitted from $\mathbf{z}$ for conciseness.

\section{EsTIMATION TECHNIQUE}

Most work on location estimation methods is based on the maximum likelihood estimator (MLE) technique [9], [17], [20]-[24]. The MLE location estimate $\hat{\boldsymbol{\theta}}_{\mathrm{MLE}}$ is calculated using

$$
\hat{\boldsymbol{\theta}}_{\mathrm{MLE}}=\arg \max _{\boldsymbol{\theta}} f_{\mathbf{Z}}(\mathbf{z} \mid \boldsymbol{\theta})
$$

where $f_{\mathbf{Z}}(\mathbf{z} \mid \boldsymbol{\theta})$ is the conditional probability density function (pdf) of the measurements given the location [25]. The estimated location vector is $\hat{\boldsymbol{\theta}}=(\hat{x}, \hat{y})$, where $\hat{x}$ is the estimated $\hat{x}$-coordinate and $\hat{y}$ is the estimated $\hat{y}$-coordinate of the mobile terminal. A subscript will be appended to $\hat{\boldsymbol{\theta}}$ to indicate the estimation algorithm. The main attraction of the MLE technique is that given propagation and noise models, it is possible to estimate the location of the mobile terminal from measurements. The MLE solution requires no prior information about the location of the mobile terminal.

In practice, however, the cellular network has some knowledge of the location of the mobile terminal [15]. The handoff algorithm determines which base station serves the mobile terminal at any given time, which provides prior statistical knowledge about the mobile terminal location. This location information is returned in Phase I of the FCC's E911 wireless location requirement [1].

The simplest estimator that makes use of prior information about location is the maximum a posteriori (MAP) estimator. The MAP estimator in its general form is given by [13]

$$
\hat{\boldsymbol{\theta}}_{\mathrm{MAP}}=\arg \underset{\boldsymbol{\theta}}{\max } f_{\boldsymbol{\Theta}}(\boldsymbol{\theta} \mid \mathbf{z})
$$

This estimator does not make optimum use of the prior knowledge of mobile terminal location. An estimator that makes better use of the prior knowledge to reduce the estimation error is presented next.

If prior information about the mobile terminal location exists, the mean square error (MSE), defined as $\mathrm{E}\left\{[\hat{\boldsymbol{\theta}}-\boldsymbol{\theta}]^{T}[\hat{\boldsymbol{\theta}}-\boldsymbol{\theta}]\right\}$ ( $E[\cdot]$ denotes the expectation operator) where the expectation is with respect to $\boldsymbol{\theta}$ and $\hat{\boldsymbol{\theta}}$, can be used as the criterion of optimality. The optimal estimator is the minimum mean square error (MMSE) estimator, which is given by

$$
\begin{aligned}
\hat{\boldsymbol{\theta}}_{\mathrm{MMSE}} & =\mathrm{E}[\boldsymbol{\theta} \mid \mathbf{z}] \\
& =\int_{S} \boldsymbol{\theta} f_{\Theta}(\boldsymbol{\theta} \mid \mathbf{z}) d \boldsymbol{\theta}
\end{aligned}
$$

where $f_{\boldsymbol{\theta}}(\boldsymbol{\theta} \mid \mathbf{z})$ is the posterior conditional pdf of location $\boldsymbol{\theta}$ given the measurement vector $\mathbf{z}$, and $\boldsymbol{S}$ is the region in which the mobile terminal is known to reside [13]. The difference between the
MMSE estimator and the MLE is made clearer by expanding the $f_{\boldsymbol{\theta}}(\boldsymbol{\theta} \mid \mathbf{z})$ pdf as follows:

$$
\begin{aligned}
\hat{\boldsymbol{\theta}}_{\mathrm{MMSE}} & =\int_{\boldsymbol{S}} \boldsymbol{\theta} \frac{f_{\mathbf{Z}, \Theta}(\mathbf{z}, \boldsymbol{\theta})}{f_{\mathbf{Z}}(\mathbf{z})} d \boldsymbol{\theta}=\frac{\int_{\boldsymbol{S}} \boldsymbol{\theta} f_{\mathbf{Z}, \Theta}(\mathbf{z}, \boldsymbol{\theta}) d \boldsymbol{\theta}}{\int_{\boldsymbol{S}} f_{\mathbf{Z}, \boldsymbol{\Theta}}(\mathbf{z}, \boldsymbol{\theta}) d \boldsymbol{\theta}} \\
& =\frac{\int_{\boldsymbol{S}} \boldsymbol{\theta} f_{\mathbf{Z}}(\mathbf{z} \mid \boldsymbol{\theta}) f_{\Theta}(\theta) d \boldsymbol{\theta}}{\int_{\boldsymbol{S}} f_{\mathbf{Z}}(\mathbf{z} \mid \boldsymbol{\theta}) f_{\boldsymbol{\Theta}}(\theta) d \boldsymbol{\theta}} .
\end{aligned}
$$

The key difference from the MLE is that the MMSE estimator uses prior knowledge of the location of the mobile terminal, i.e., the pdf $f_{\Theta}(\boldsymbol{\theta})$, to improve the location accuracy.

The conditional pdf $f_{\mathbf{Z}}(\mathbf{z} \mid \boldsymbol{\theta})$ is dependent on the probability of NLOS propagation and LOS propagation at location $\boldsymbol{\theta}$ and the conditional pdf of excess delay if NLOS propagation is occurring, both of which are unknown prior to location estimation [12]. The solution in the previous literature is to use an assumed conditional pdf. The accuracy of the location estimates is dependent on the accuracy of the assumed conditional pdf and thus the validity of the assumed propagation model. In the literature on MLE location estimation, the propagation model used is LOS propagation and the measurement noise vector $\mathbf{v}$ is assumed to be a Gaussian vector with a covariance matrix equal to $\mathbf{C}$ defined in (6) [9], [17].

To implement the MMSE estimator in (10), the joint pdf of $\mathbf{z}$ and $\boldsymbol{\theta}$ is needed. This is equivalent to knowledge of the conditional pdf of the measurement vector $\mathbf{z}$ given location $\boldsymbol{\theta}$, discussed in the previous paragraph, and also knowledge of the marginal pdf of the location $f_{\Theta}(\boldsymbol{\theta})$.

This paper proposes the generation of the assumed pdf from data collected from the location environment. There are two methods of estimating the pdf from data: parametric and nonparametric.

The parametric technique calculates the estimated joint pdf as

$$
\hat{f}_{\mathbf{Z}, \Theta}(\mathbf{z}, \boldsymbol{\theta})=g\left(\mathbf{z}, \boldsymbol{\theta}, p_{1}, p_{2}, \ldots, p_{n}\right)
$$

with $p_{1}, p_{2}, \ldots, p_{n}$ being parameters of the probability density function. The values of $p_{j}$ would be calculated based on models of the propagation environment and survey measurements. For example, if $\mathbf{z}$ and $\boldsymbol{\theta}$ were jointly Gaussian, then the parameters would be their means and covariances for which methods of estimation from data are well known. The difficulty in location estimation is that simple parametric models of the propagation environments only exist except for LOS propagation cases [26]. Dealing with NLOS propagation cases is much more complex. The tools that do exist for NLOS modeling use ray-tracing to estimate the propagation conditions at predetermined points within the environment but do not provide parametric models for variations of the attributes of interest over regions [27]-[29].

The basic idea of nonparametric estimation is, from a given set of data, to create an approximation of the pdf that created the data. This approximate pdf is a sum of kernel functions. Each component function is centered at a single survey measurement, and its value decreases monotonically as the distance from its central point increases. Thus points close to several survey points will have higher approximate pdf values than points far away from survey points. With a good selection of kernel functions, this technique can create good approximations of the pdf. 
For the location estimation problem, the survey set for a region $\boldsymbol{S}$ consists of measurements $\left\{\mathbf{z}_{\mathbf{1}}, \mathbf{z}_{\mathbf{2}}, \ldots, \mathbf{z}_{\mathbf{n}}\right\}$ made at respective true locations $\left\{\boldsymbol{\theta}_{1}, \boldsymbol{\theta}_{2}, \ldots, \boldsymbol{\theta}_{n}\right\}$. Optimally, the pdf of survey point locations is the prior pdf of mobile terminal location before location measurements are made. In the absence of any other information, a uniform density function over $\boldsymbol{S}$, the known region of mobile terminal residence, is used, as this is the prior density with the highest relative entropy [13]. The propagation survey data can be obtained from field measurements or generated using computer ray-tracing propagation models.

In general nonparametric estimation, the kernel function for survey point $j$ is a function of the location of the survey point $\boldsymbol{\theta}_{j}$ and the measurement at that survey point $\mathbf{z}_{\mathbf{j}}$. A simple estimator with good performance is created if this kernel function is the product of separate kernel functions for the location $\boldsymbol{\theta}_{j}$ and measurement $\mathbf{z}_{\mathbf{j}}$ [30]. This results in an approximate density function that is very simple to manipulate. We will designate the kernel functions for the measurements as $K_{z}(\mathbf{z})$ and kernel function for the locations as $K_{\theta}(\boldsymbol{\theta})$. The joint pdf of locations and measurements is approximated as a sum of the product of the kernel functions for each survey measurement [31]

$\hat{f}_{\boldsymbol{\Theta}, \mathbf{Z}}(\boldsymbol{\theta}, \mathbf{z})=\frac{1}{n} \sum_{j=1}^{n}\left(h_{z}\right)^{-t}\left(h_{\theta}\right)^{-2} K_{z}\left(\frac{\mathbf{z}-\mathbf{z}_{\mathbf{j}}}{h_{z}}\right) K_{\theta}\left(\frac{\boldsymbol{\theta}-\boldsymbol{\theta}_{j}}{h_{\theta}}\right)$.

The constant $l$ is the length of the measurement vector $(l=$ $k$ ) for ToA location and $(l=k-1)$ for TDoA location. The smoothing constants $h_{z}$ and $h_{\theta}$ determine the width of each of the kernel functions. For simplicity, one usually chooses kernel functions that are multivariate probability density functions with zero mean [30].

If we substitute the estimated probability density from (12) into the MMSE equation (10) and perform the integrations, the resulting estimated location is a weighted averaged of the survey point positions with the weights being determined by the measurement data (the full details of the derivation of the weighted average estimator are provided in [31] as follows:

$$
\hat{\boldsymbol{\theta}}_{\mathrm{MMSE}}=\sum_{j=1}^{n} \boldsymbol{\theta}_{j} w_{j}(\mathbf{z})
$$

where

$$
w_{j}(\mathbf{z})=\frac{K_{z}\left(\frac{\mathbf{z}-\mathbf{z}_{\mathbf{j}}}{h_{z}}\right)}{\sum_{m=1}^{n} K_{z}\left(\frac{\mathbf{z}-\mathbf{z}_{\mathbf{m}}}{h_{z}}\right)} .
$$

Using the survey data, it is possible to create a nonparametric version of the MAP estimator. Assuming that the noise probability density function has zero mean and decreases monotonically for noise values farther from the mean, the nonparametric MAP estimator is given by

$$
\begin{aligned}
\left\|\mathbf{z}-\mathbf{z}_{\mathbf{j}}\right\|^{2} & =\min \left\|\mathbf{z}-\mathbf{z}_{\mathbf{i}}\right\|^{2} \forall i \in\{1,2, \ldots, n\} \\
\rightarrow \hat{\boldsymbol{\theta}}_{\mathrm{MAP}} & =\boldsymbol{\theta}_{j}
\end{aligned}
$$

where $\|\cdot\|^{2}$ specifies the Euclidean distance. This approximate estimator approaches the true MAP estimator as $n \rightarrow \infty$.

The performance of the nonparametric location estimators is dependent on 1) selection of $\left.K_{z}(\cdot), 2\right)$ the value of the smoothing constant for the measurement vectors $h_{z}$ and the
TABLE I

KERNEL FUNCTIONS

\begin{tabular}{c|c}
\hline Kernel name & Kernel function $K(\mathbf{x})$ \\
\hline Parzen Gaussian [30,32] & $\left(\frac{1}{\sqrt{2 \pi}}\right)^{k}|\mathbf{C}|^{-\frac{1}{2}} \exp \left(-\frac{\mathbf{x}^{T} \mathbf{C}^{-1} \mathbf{x}}{2}\right)$ \\
\hline Parzen Laplace [32] & $\frac{1}{2} \exp \left(-\|\mathbf{x}\|^{1}\right)$ \\
\hline & $\prod_{j=1}^{k} \frac{1}{\pi} \frac{1}{1+\left(\mathbf{x}_{j}\right)^{2}}$ \\
\hline Distance based [33] & \\
\hline
\end{tabular}

number of survey points $n$, and 3) the distribution of survey point locations. Each of these will be discussed in order below.

The kernel functions used in this paper are listed in Table I. The matrix $\mathbf{C}$ used in the Parzen Gaussian kernel is the covariance matrix $\mathbf{C}$ defined in (6). These kernel functions are selected because of good performance when applied to estimation in similar problem domains [30], [31].

Selecting optimal values for $n$ and $h_{z}$ is a difficult task, made more difficult since the optimal value of each is dependent on the value of the other. Larger values of $h_{z}$ result in each survey point having a larger region of influence in the sample space for the estimated density. Small values of $h_{z}$ mean that the influence region of each survey point is small with the estimated density function becoming a sum of delta functions as $h_{z} \rightarrow 0$.

The optimal values of $h_{z}$ and $n$ for each kernel are a function of the actual density function being estimated and are thus unknown for any given estimation problem. It is, however, known that using values of $h_{z}$ and $n$ that have the correct order of magnitude allows one to obtain results almost as good as using those obtained using the optimal value in many cases [30].

An examination of the kernel functions in Table I and (12) reveals that $h_{z}$ has some relation to the standard deviation of the measurement noise. For example, when the Parzen window Gaussian kernel from Table I is used, $h_{z}$ goes in the place where the standard deviation appears in the Gaussian density function. Therefore, it appears that $h_{z}$ is of the same order of magnitude as the standard deviation of the measurement noise. In Section V, the relative insensitivity of the estimation technique to variations in the value of $h_{z}$ will be shown.

The number of survey points $n$ is a critical factor on the performance of the system. The mean distance error $\mathrm{E}\left[\sqrt{(x-\hat{x})^{2}+(y-\hat{y})^{2}}\right]$, where $(x, y)$ is the location of the mobile terminal and $(\hat{x}, \hat{y})$ is the estimated location of the mobile terminal, can be viewed as a distance. Survey points that are separated by a distance less than the mean distance error cannot be reliably differentiated by a location estimator. If the survey set does not have measurement noise, then survey points that are separated by a distance less than the mean distance error add redundant information to the survey set. 
This suggests that a heuristic for the number of survey points needed within a region $\boldsymbol{S}$ is to divide the area of $\boldsymbol{S}$ by $\pi$ times the squared mean distance error. This assigns each survey point an area equal to the area of a disc with a radius equal to the mean distance error. Unfortunately, the mean distance error is not known for a given location scenario. A solution to this dilemma is to replace the mean distance error in the formula for $n$ with a lower bound on the root-mean-squared error (RMSE) $\sqrt{\mathrm{E}\left[(x-\hat{x})^{2}+(y-\hat{y})^{2}\right]}$, which can be calculated. This gives an estimate for the value of $n$ for the optimal value of $h_{z}$. Since other values of $h_{z}$ will result in higher RMSE and thus need fewer survey points, use of the lower bound on RMSE gives an approximate upper bound on $n$. The lower bound on the RMSE can be calculated using the Weinstein-Weiss lower bound described in the Appendix. This bound takes into consideration both the prior pdf of location and the effects of NLOS propagation.

We will now consider the effect of measurement noise on the required number of survey points. Measurement noise in the survey set measurements means that the survey set has imperfect knowledge of the propagation environment. Increasing $n$ will reduce the effect of measurement noise in the survey data on estimation error as the effect of measurement noise is averaged out. Using the Weak Law of Large Numbers [34], it can be shown that as $n \rightarrow \infty$, the survey set gives perfect information about the mean value of the measurement vector at all points in the region $\boldsymbol{S}$. Fortunately, our simulation results in Section V show that generally $n$ only needs to be increased by a factor of one to four to reduce the effect of survey set measurement noise to acceptable levels.

The prior pdf of $\boldsymbol{\theta}$ contains information from the handoff algorithm, as mentioned in the Introduction, and the selection of base stations whose measurements are used to locate the mobile terminal. In terms of nonparametric estimation, the prior pdf of location is equivalent to the problem of choosing the subset of all collected survey points that will be used to locate a given mobile terminal. We will first address the problem of base-station selection and then discuss how the result of this selection is used for survey point selection.

Which base stations are selected to estimate a mobile terminal's position can affect the accuracy of the results. This so-called geometric dilution of precision (GDOP) refers to the ratio of root mean square position error to root mean square ranging error [35]. The GDOP ratio is dependent on the relative position of the mobile terminal to the base stations. The GDOP describes the phenomena of high location estimation error when the mobile terminal is within certain regions [36].

The problem is worse for TDoA estimation than for ToA location estimation. The root cause of this is that the propagation time difference measurements used in TDoA location estimation contain less information than the time measurements used in ToA location estimation.

The use of MMSE-based estimation techniques reduces the GDOP problem. Knowledge of the prior pdf of $\boldsymbol{\theta}$ allows possible ambiguous location estimates to be removed from consideration.

Optimally, one would like to select base stations that surround the mobile terminal. That is, if a polygon is formed by drawing lines connecting the base stations' positions without crossing, then the mobile terminal position should be located in the polygon's interior. If the mobile terminal is located outside of this polygon, then GDOP effects can increase the estimation error. This phenomenon is described in [35] and [36].

Using the prior information about mobile terminal position from handoff data, it is possible to reduce bad base-station selection by using the prior location information to make sure that the base stations selected describe a polygon that encloses as much of the known region of residence as possible. Reduction of GDOP is not the only consideration for base-station selection. Due to restrictions on maximum transmission power and interference that a base station is allowed to generate in surrounding cells, the maximum distance between a base station and mobile terminal that it can communicate with is limited. Therefore, the number of base stations that can possibly make measurements to locate a given mobile terminal is limited. Furthermore, the information that can be used to make the base station selection is also restricted.

Others [9] have assumed that the closest base stations are used, but such a policy provides the estimator with information that would not be available in the field. If a network has perfect knowledge of which base stations are closest to a mobile terminal and knowledge of all base-station positions, regions can be deterministically removed from $\boldsymbol{S}$. In other words, if we know that one base station is selected while a second base station is not, all survey points in $\boldsymbol{S}$ closer to the second base station than the first base station can be excluded from the position estimation calculation.

In actual cellular networks, each serving base station has a handoff set consisting of all of its neighboring base stations. We will assume that a mobile terminal is served by the base station with the lowest distance measurement. The base-station selection method used in this paper is to use the currently serving base station plus the $k-1$ base stations out of the handoff set with the lowest distance measurements to locate the mobile terminal for a total of $k$ base-station measurements. This method has the advantage that only information available to the mobile terminal is required to make the base-station selection. Additionally, by restricting base-station selections to only surrounding cells, the probability that the mobile terminal will be surrounded by the base stations is high, reducing GDOP effects.

The system does not have perfect knowledge of mobile terminal cell residency. For this reason, we use survey points in the cell of the serving base station plus some survey points from adjacent cells to locate the mobile terminal. For the simulations reported later in this paper, the survey points used to locate the mobile terminal are all survey points within a distance of 1.25 cell radii from the base station with the smallest propagation distance measurement. The optimum distance into other cells that the survey set should extend is a function of the measurement noise. If the measurement noise is high, the mobile is more likely to be in a cell adjacent to the cell of the serving base station so more survey points from adjacent cells are required. The values of 1.25 was selected, as it gave reasonable performance at relatively low additional cost for a wide range of value for $\sigma$.

It is possible with the measurement-based base-station selection method to calculate a revised location pdf $f_{\Theta}(\theta)$, where the 
density function value at a point $\theta$ is proportional to the probability that the selected base stations will have the lowest measured distance if the mobile terminal is located at that point. Unfortunately, this function's value cannot be calculated within the real-time constraints of a mobile terminal location system. The survey points in which the $k$ base stations selected for location measurements by the mobile terminal location process have the lowest surveyed distance measurements can be used to create an approximate prior probability density function for the mobile terminal location. For our nonparametric kernel-based estimators, this prior function can be included by only using the survey points in our calculation that are likely to have high calculated values from the prior location probability density function. The survey point selection process is to only include survey points in which the set of $k+m$ base stations with the lowest measured propagation distances includes the $k$ base stations that were selected by the location process. Only these survey points are used in the calculation of (13) and (14). The optimal value of $m$ is dependent on the cell layout, with $m$ being higher for cases where more cells border each other. For cases where six cells border the current cell, the standard hexagonal cell case, or where four cells border the current cell, an urban grid of cells, a value for $m$ of one or two gives good results. This gives an estimation algorithm with lower computational cost, where only $N$ survey points satisfying the selection conditions are used instead of all $n$ points in the survey set.

\section{SIMULATION DESCRIPTION}

The estimators are evaluated for location accuracy when the mobile terminal is located outdoors in urban microcells, since this is the region of greatest interest to cellular network providers. No good model exists for propagation delay measurements for indoor locations. It can be assumed that indoor locations will have mostly NLOS propagation and incur large path-loss penalties from having to penetrate building windows and/or walls [37]. These attributes can be used to differentiate indoor and outdoor measurement vectors, so if indoor survey points were added to the survey set, it would not reduce the location accuracy.

A square microcell with sides of length $300 \sqrt{2}$ m was used to evaluate the estimator accuracy. The geometry of the base-station configuration for a single cell is shown in Fig. 1. The hatched regions represent buildings. This configuration was used since it has been used to evaluate other mobile terminal location schemes [9]. The central cell and the two rings of cells around it are included in the simulations. The mobile terminal location is sampled from a uniform distribution over the central cell's street locations. This is the worst case when there is no prior information about mobile terminal location other than cell residence and the mobile terminal is located outdoors since the entropy of the mobile location distribution is maximized.

When the LOS path between a base station and mobile terminal is unobstructed, the propagation distance is simply the Euclidean distance between the mobile terminal and base station. For NLOS propagation, the radio signal is assumed to diffract around the corners of buildings. The propagation path is thus the distance from the base station to the corner plus the distance from the corner to the mobile terminal. This propagation distance is $d_{c}+d_{r}$, as shown in Fig. 1 .

Measurement noise is modeled as the sum of two independent noise sources. The first source is a zero-mean Gaussian random vector with a covariance matrix of $\sigma^{2}$ times an identity matrix representing the error in the synchronization in the mobile terminal's receiver [38]. The second noise source represents small scatterers such as people, vehicles, and foliage. The value of this delay is the smallest value of 20 independent draws from a uniform distribution from 0-720 m. This matches the lowest excess propagation delay when the channel is modeled using the "urban" propagation model from [39]. The measurement noise for each of the base station's measured propagation distance vector defined in (2) is independently and identically distributed for all base stations. The measurement noise is also independent from generation of the survey set to generation of the measurement vectors.

Measurements from three base stations are used to locate the mobile terminal, $k=3$. The method described in the previous section is used to select the measuring base stations from a set of possible base stations consisting of the base station with the lowest distance measurements and its eight closest neighbors. For TDoA measurement data, the base station with the lowest distance measurement is the reference base station. Only those survey points where the three selected measuring base stations are in the set of the five base stations with the lowest measurements are used to locate the mobile terminal. This is the survey point selection algorithm with $m=2$ described in the preceding section.

The figure of merit used to judge performance is the RMSE defined as the square root of the sum of the mean squared error for each of the estimated coordinate values.

The objective of the simulations is to evaluate the performance of the nonparametric kernel-based estimators and show that these estimators are robust to variations of the parameters of the kernel functions and the environment.

The first set of simulations was performed with 100 survey points, $n=100$. The survey points are uniformly distributed over street locations so that there are $n$ survey points within a distance of 1.25 cell radii of each base station. A uniform density is used since this is the density with the highest relative entropy representing the prior probability density function with the least amount of information. The value of $h_{z}$ was varied to see how robust the estimators are to variations of this parameter.

The second set of simulations was performed to show the robustness of the kernel estimators to different sizes of the survey set. The number of survey points $n$ was varied from ten to 100 , and the RMSE of the different estimators was recorded.

The third set of simulations was performed to measure the performance at different levels of measurement noise. The standard deviation of the Gaussian measurement noise $\sigma$ is varied from 15 to $50 \mathrm{~m}$ and the RMSE recorded. The simulations are performed with random survey point locations and survey point locations arranged in a regular deterministic pattern to see if the latter will alter performance. For the deterministic pattern, the survey points are placed in two perpendicular lines down the center of the vertical and horizontal streets with regular spacing. A lower bound on the RMSE for location estimation error was 


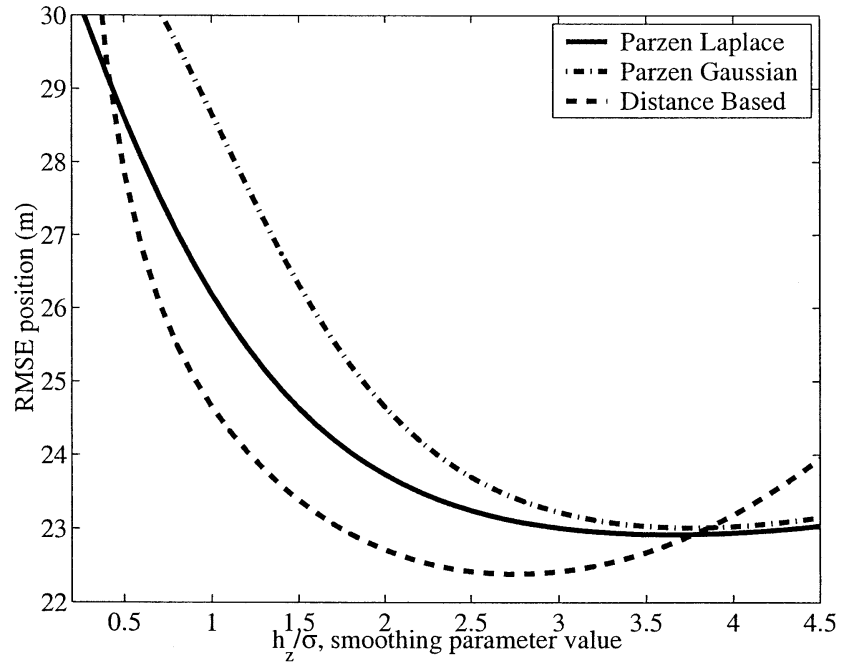

Fig. 2. ToA estimator performance for differing $h_{z}$ values $(n=100, \sigma=$ $15 \mathrm{~m})$.

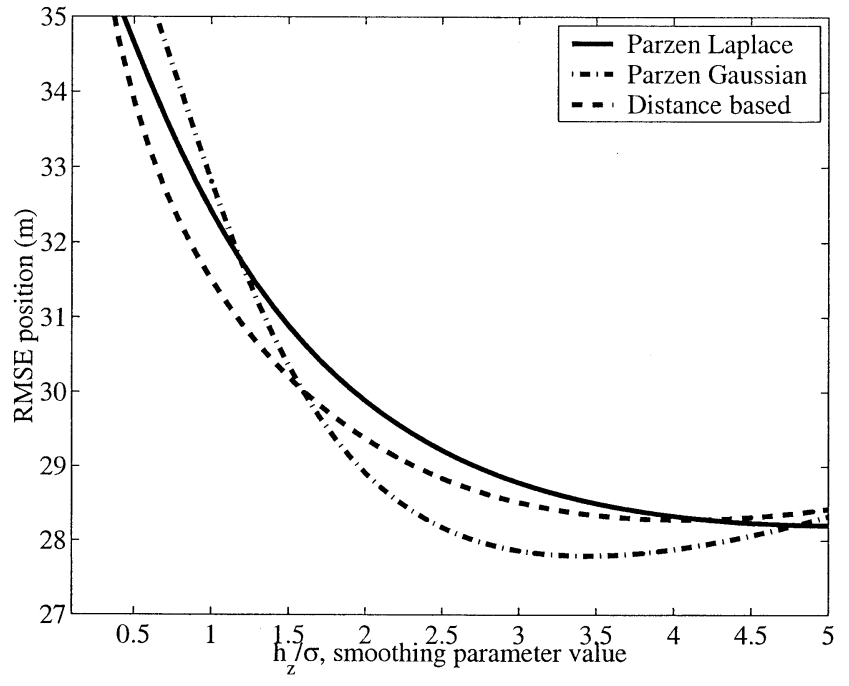

Fig. 3. TDoA estimator performance for differing $h_{z}$ values $(n=100, \sigma=$ $15 \mathrm{~m})$.

also calculated to see how close the estimator comes to optimal performance.

The Weinstein-Weiss lower bound was used to bound the estimator's performance. It gives a lower bound on the MSE of a parametric estimator [40] when the prior distribution of the parameter to be estimated is known. The true prior probability density function for the mobile terminal location given the set of base stations selected for measurements in the simulations is, as described above, a function of the base-station selection algorithm and is difficult to derive. We use a prior probability density function that is more informative than the true prior density in the Weinstein-Weiss lower bound calculation. The extra information in this prior ensures that the lower bound calculated is lower than the lower bound calculated using the true prior density. Thus, the RMSE lower bound calculated is guaranteed to be lower than the RMSE from the simulations allowing for valid comparisons. The true propagation distances used in the Weinstein-Weiss calculations take into account NLOS effects from the buildings. The propagation distance for a given base station
TABLE II

OPTIMAL SMOOTHING PARAMETER FOR $\sigma=15.0 \mathrm{~m}$

\begin{tabular}{l|l|l}
\hline Kernel function & ToA Optimal $h_{z}$ & TDoA Optimal $h_{z}$ \\
\hline Parzen Laplace & $3.6 \sigma$ & $5.0 \sigma$ \\
\hline Parzen Gaussian & $3.8 \sigma$ & $3.4 \sigma$ \\
\hline Distance based & $2.7 \sigma$ & $4.0 \sigma$ \\
\hline
\end{tabular}

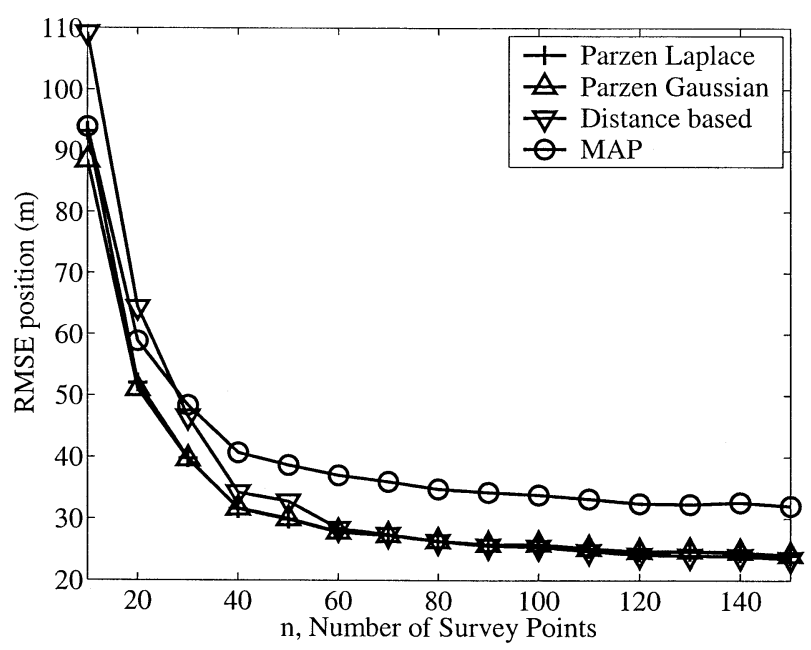

Fig. 4. ToA estimator performance for differing $n$ values $(\sigma=15 \mathrm{~m})$.

and the mobile terminal is the true distance between them when the propagation is LOS and is equal to $d_{c}+d_{r}$, as shown in Fig. 1 when the propagation is NLOS. Only the Gaussian measurement noise is considered in the Weinstein-Weiss bound calculations. No estimator can have lower RMSE than the value the lower bound indicates. The RMSE of the simulated estimators relative to the lower bound indicates how well the estimators make use of the measurement and prior information. The computation of the bound is described in the Appendix.

\section{RESULTS}

The robustness of the nonparametric estimators to variations of the smoothing parameter $h_{z}$ is demonstrated in the first set of simulations. The standard deviation of the measurement noise was $15 \mathrm{~m}$, and 100 survey points were generated $(\sigma=15$, $n=100)$. The plot of RMSE versus $h_{z}$ value for the ToA estimator is shown in Fig. 2. The results for the TDoA estimator are shown in Fig. 3. The optimal values of $h_{z}$ are shown in Table II. The ToA estimators gave better performance than the TDoA estimators. For all estimators and kernel functions, the impact of using values of $h_{z}$ greater than the optimum value was less than that of using values of $h_{z}$ less than the optimum value. The results show that the value of $h_{z}$ only needs to be within the same order of magnitude as the optimum value for good results. For the following simulations, the optimum value of $h_{z}$ is used for each kernel estimator.

The second set of simulations shows the results for variations of the number of survey points. The results for different values of $n$ are shown in Fig. 4 for the ToA estimator and Fig. 5 for the 


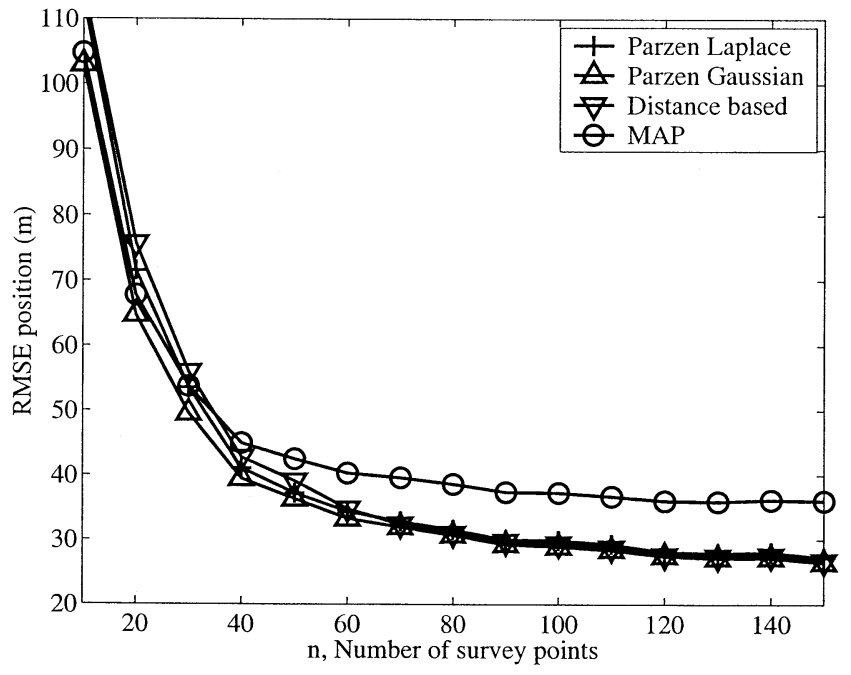

Fig. 5. TDoA estimator performance for differing $n$ values $(\sigma=15 \mathrm{~m})$.

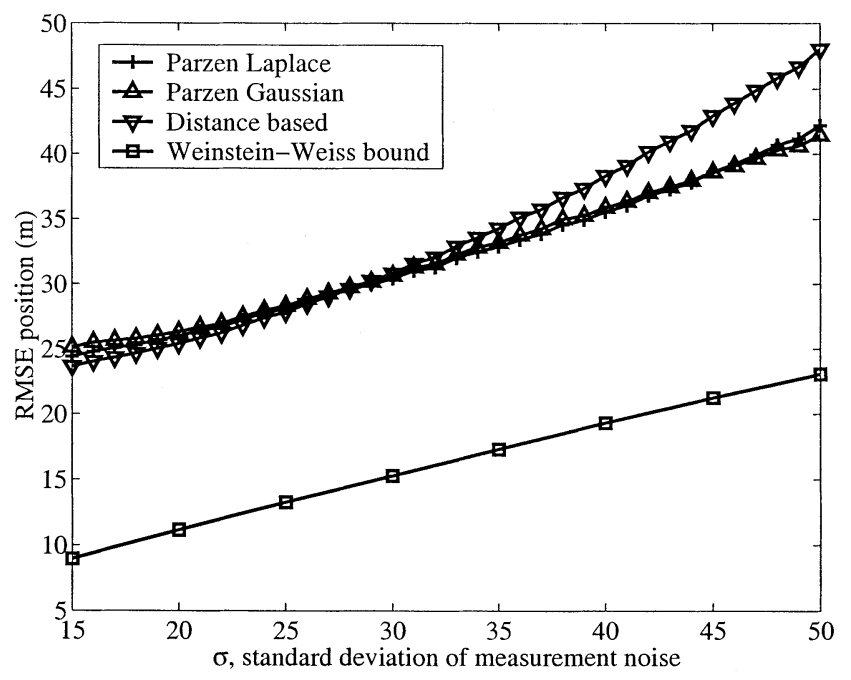

Fig. 6. ToA estimator performance for differing $\sigma$ values $(n=100)$.

TDoA estimator. Once the survey set reaches a certain size, new survey points are adding mostly redundant information. This point appears to be around $n=60$ for both the ToA and TDoA estimators. The decision of how many survey points are needed is a function of the cost of taking the survey measurements and how much accuracy is desired. However, the designer should remember that there is a limit to MSE accuracy determined by the measurement noise. This limit is discussed in the Appendix. Using the heuristic for calculating $n$ described in Section III, with lower bound on the MSE for $\sigma=10 \mathrm{~m}$ and ToA location (see Fig. 6) calculated using the Weinstein-Weiss bound of $100 \mathrm{~m}^{2}$ and a street area in each cell of $(290 \cdot 20)+(20 \cdot 20)=$ $23600 \mathrm{~m}^{2}$, we get an estimate of $n \approx 23600 /(\pi \cdot 100)=75.12$ points in a cell. The ToA estimators perform slightly better than the TDoA estimators. The reason for this is the information reduction of TDoA with respect to ToA estimators. For all of the following simulation results, $n=100$.

The third set of simulations measured the performance of the estimators as the standard deviation of the Gaussian component of the measurement noise $\sigma$ was varied. The results for ToA

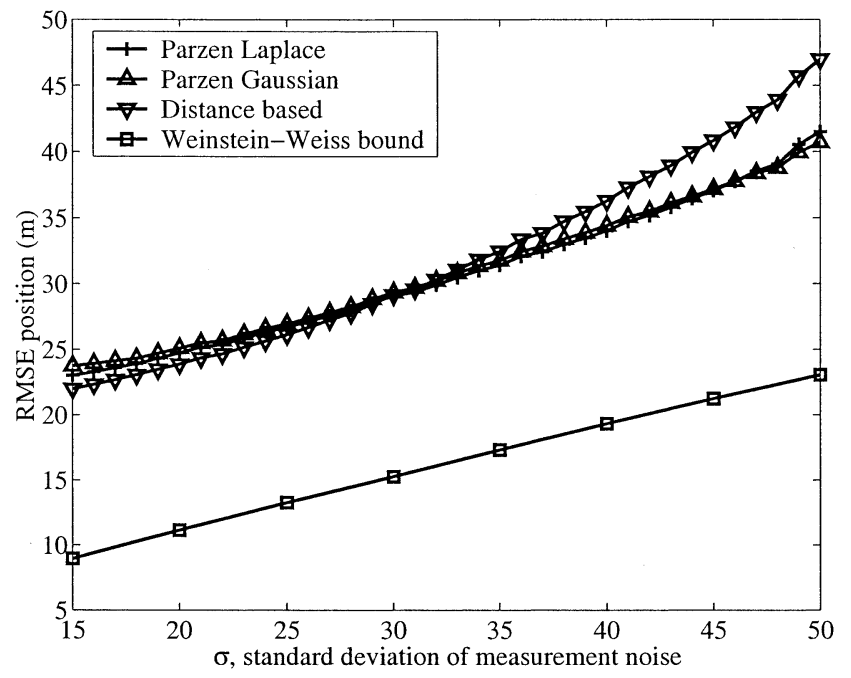

Fig. 7. ToA estimator performance for differing $\sigma$ values (deterministic survey point locations, $n=100$ ).

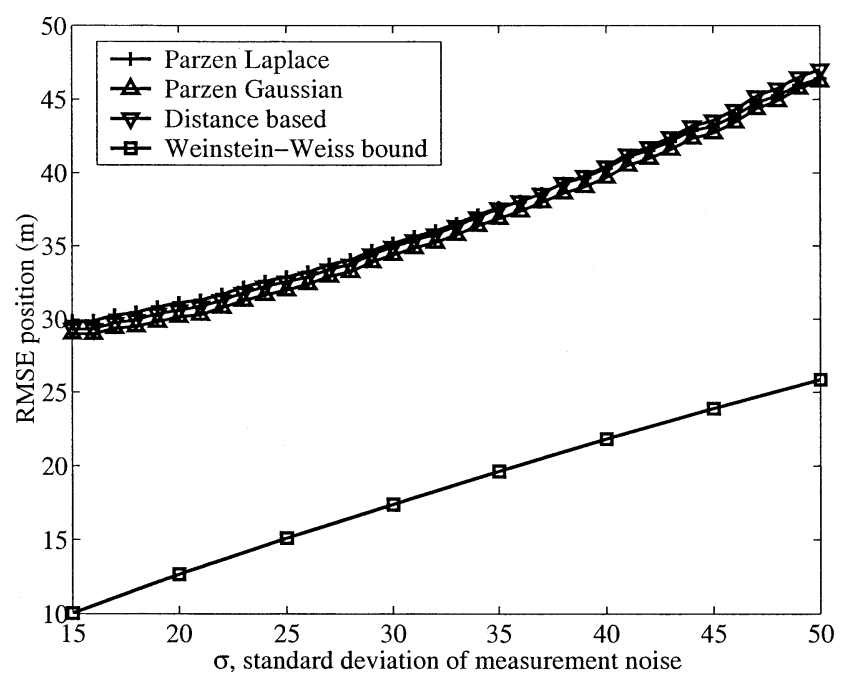

Fig. 8. TDoA estimator performance for differing $\sigma$ values $(n=100)$.

and TDoA simulations with random survey point locations are shown in Figs. 6 and 8. The next set of simulations is performed with the deterministic regular positions of survey points. The results are shown in Fig. 7 for ToA estimators and Fig. 9 for TDoA estimators. A comparison of these results with the results for random positions of survey points shows that the accuracy of kernel estimators is not greatly affected by variations of survey point placement. For both deterministic and random survey point locations, the estimators are robust to variations of the intensity of measurement noise. The estimators perform slightly better when the survey points are in the regular deterministic pattern than with random placements, but the improvement in accuracy was only a few meters. This indicates that regularly spaced survey points, such as those likely to be collected by an automatic propagation survey method, will result in good performance for mobile terminal location.

The last set of simulations compares the accuracy of the kernel estimators against the accuracy of nonparametric MAP estimators and parametric MLE estimators. The results are graphed on Fig. 10 for a range of measurement noise variances. 


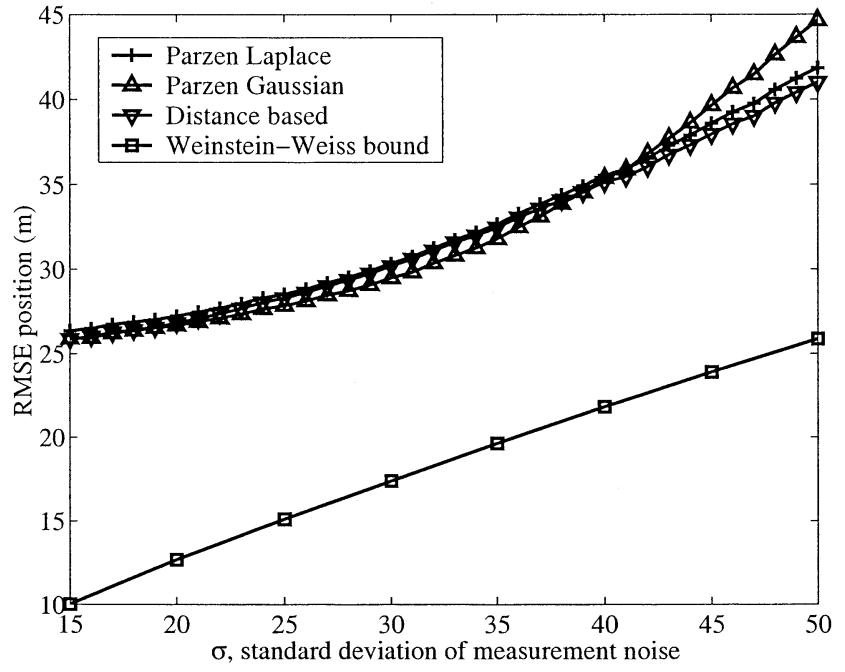

Fig. 9. TDoA estimator performance for differing $\sigma$ values (deterministic survey point locations, $n=100$ ).

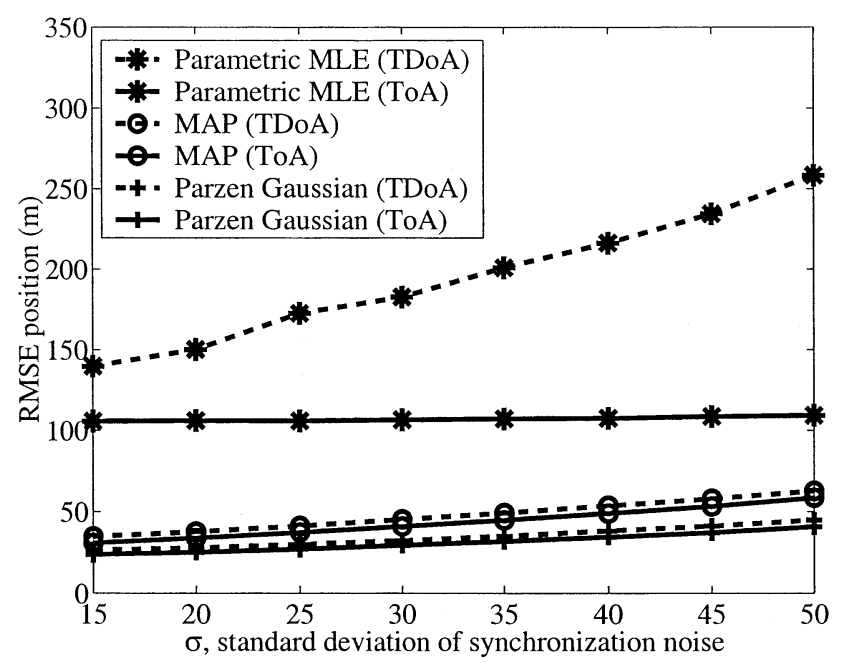

Fig. 10. Comparison of parametric and nonparametric estimators.

The parametric MLE estimators assume LOS propagation models, and the estimates are calculated using iterative solving techniques [9], [17]. The parametric estimators assume a Gaussian noise model and are given perfect knowledge of the noise variance. The ToA estimators perform better than the TDoA estimators, with the nonparametric kernel estimators giving the best performance. The nonparametric MAP estimators give the next best set of performance figures, with the parametric estimators giving the worst performance.

The Weinstein-Weiss bounds show that the nonparametric estimators give results within an order of magnitude of the best possible values. The ToA estimators perform better than the TDoA estimators. Since the Weinstein-Weiss bounds are calculated for an estimator with a more informative prior probability density function for the location than the simulated estimators and without the additional small scatterer noise, the results show that the nonparametric estimators give very attractive performance values.

A seemingly plausible argument could be made that the poor performance of the MLE estimators is a result of assuming the
LOS propagation model. Indeed, the performance of the MLE estimators can be improved if the estimators have greater knowledge of the propagation model. If an accurate parametric model of the propagation environment exists, then the optimal estimator is the MMSE estimator, not the improved MLE estimator. However, usually a parametric model does not exist, so the nonparametric techniques described in this paper are proposed.

\section{CONCLUSION}

This paper has introduced the use of nonparametric kernelbased estimators for location of mobile terminals in wireless networks using measurements of propagation delays. It has been demonstrated that these estimators perform better than the previously used parametric MLE estimators for the case of a simulated microcell environment with NLOS radio propagation. These estimators were shown to give excellent results at several different levels of measurement noise. Methods for calculating good values for parameters of the kernel functions were demonstrated, as well as the robustness of the estimators when the values of the parameters vary from the optimal points.

The performance of ToA and TDoA location estimators is very close, with the ToA consistently outperforming TDoA. This result is not surprising, given that TDoA measurements can be derived from ToA but not vice versa, indicating that ToA measurements contain more information than TDoA measurements. The nonparametric estimators are compared with a Weinstein-Weiss lower bound on estimator performance. This comparison shows that the ToA and TDoA estimators have accuracy comparable to the lower bound and are thus fairly efficient.

\section{APPENDIX \\ WEINSTEIN-WEISS LOWER BOUND ON MSE FOR LOCATION ESTIMATES}

It is desirable when estimating a parameter to have a lower bound on the estimation error. This allows the estimation designer to judge the performance of their estimator. A popular lower bound in location estimation is the Cramer-Rao lower bound [11], [41]. (It should be noted that [42] derives a bound that is equivalent to the Cramer-Rao bound.) These bounds are local bounds: they give bounds on the estimation error for $\hat{\theta}$ when $\boldsymbol{\theta}$ is a fixed known value.

There are two problems with existing lower bounds. First, the derived bounds also assume that the estimator is locally unbiased, that is, $\mathrm{E}[\hat{\boldsymbol{\theta}} \mid \boldsymbol{\theta}]=\boldsymbol{\theta}$ for all $\boldsymbol{\theta}$. It has been shown that any MMSE estimator is biased [43], and the GDOP can cause large bias in location estimation with MLE estimators [35]. Second, the bounds do not take into account the known prior probability density functions for $\boldsymbol{\theta}$.

There is an extension of the Cramer-Rao lower bound-the Bayesian Cramer-Rao lower bound, described in [43] — which incorporates the prior probability density for $\boldsymbol{\theta}$ with the Cramer-Rao bound to calculate a bound on the MSE. This bound does require that the estimator be locally unbiased. One of the conditions for the Bayesian Cramer-Rao bound to be valid for location estimation is that $\left(\partial f_{\mathbf{Z}, \boldsymbol{\Theta}}(\mathbf{z}, \boldsymbol{\theta})\right) /(\partial x),\left(\partial f_{\mathbf{Z}, \boldsymbol{\Theta}}(\mathbf{z}, \boldsymbol{\theta})\right) /(\partial x),\left(\partial f_{\mathbf{Z}, \boldsymbol{\Theta}}(\mathbf{z}, \boldsymbol{\theta})\right) /(\partial y)$, 
$\left(\partial^{2} f_{\mathbf{Z}, \boldsymbol{\Theta}}(\mathbf{z}, \boldsymbol{\theta})\right) /\left(\partial x^{2}\right)$,

$\left(\partial^{2} f_{\mathbf{Z}, \Theta}(\mathbf{z}, \boldsymbol{\theta})\right) /\left(\partial y^{2}\right)$

and $\left(\partial^{2} f_{\mathbf{z}, \boldsymbol{\Theta}}(\mathbf{z}, \boldsymbol{\theta})\right) /(\partial x \partial y)$ be absolutely integrable with respect to $x, y$, and $\mathbf{z}$ [43]. Unfortunately, the points of transition between LOS and NLOS propagation create discontinuities in the joint probability density function that cause this condition to be violated, making the Bayesian Cramer-Rao lower bound invalid for this application.

We propose the use of the Weinstein-Weiss bound on MSE, which does not have the stringent requirements of the Bayesian Cramer-Rao lower bound and also takes into account the prior known probability density function of $\boldsymbol{\theta}$ [40]. We define a function $L^{s}(\mathbf{z}, \boldsymbol{\theta}+\mathbf{h}, \boldsymbol{\theta})$ as

$$
L^{s}(\mathbf{z}, \boldsymbol{\theta}+\mathbf{h}, \boldsymbol{\theta})=\left[\frac{f_{\mathbf{Z}, \boldsymbol{\Theta}}(\mathbf{z}, \boldsymbol{\theta}+\mathbf{h})}{f_{\mathbf{Z}, \boldsymbol{\Theta}}(\mathbf{z}, \boldsymbol{\theta})}\right]^{s} .
$$

It has been demonstrated that for any vector function $\mathbf{e}(\mathbf{z})$

$$
\mathrm{E}\left\{[\boldsymbol{\theta}-\mathbf{e}(\mathbf{z})][\boldsymbol{\theta}-\mathbf{e}(\mathbf{z})]^{T}\right\} \geq \mathbf{H G}^{-1} \mathbf{H}^{T}
$$

with $\mathbf{G}$ being the $2 \times 2$ matrix with elements given by (18) and (19) as shown at the bottom of the page for any vectors $\left(\mathbf{h}_{1}, \mathbf{h}_{2}\right)$ and scalars $\left(s_{1}, s_{2}\right)$. The tightest Weinstein-Weiss lower bound is achieved by calculating the values of $s_{1}, s_{2}, \mathbf{h}_{1}$, and $\mathbf{h}_{2}$ that maximize the right-hand side of (17), although this is a difficult and computationally expensive task. A good bound can be found with $s_{1}=s_{2}=(1 / 2)$ and

$$
\mathbf{H}=h \cdot\left[\begin{array}{ll}
1 & 0 \\
0 & 1
\end{array}\right] .
$$

The elements of $\mathbf{G}$ can be rewritten as

$$
\begin{aligned}
\mathbf{G}_{i j} & =2 \frac{\mu\left(\frac{1}{2}, \mathbf{h}_{i}-\mathbf{h}_{j}\right)-\mu\left(\frac{1}{2}, \mathbf{h}_{i}+\mathbf{h}_{j}\right)}{\mu\left(\frac{1}{2}, \mathbf{h}_{i}\right) \mu\left(\frac{1}{2}, \mathbf{h}_{j}\right)} \\
\mu(s, \mathbf{h}) & =\mathrm{E}\left[L^{s}(\mathbf{z}, \boldsymbol{\theta}+\mathbf{h}, \boldsymbol{\theta})\right] .
\end{aligned}
$$

A tight bound can be found by determining the value $h$ that maximizes (17) with the other variables set as above. The limit of this case for $h \rightarrow 0$ is identical to the Bayesian Cramer-Rao lower bound [43].

To simplify the following derivations, we will denote the Mahalanobis quadratic distance function [44] as $\|\mathbf{X}\|_{\mathbf{C}^{-1}}^{2}=\mathbf{X}^{T} \mathbf{C}^{-1} \mathbf{X}$. We will denote the expected value of the measurement vector $\mathbf{z}$ when the mobile terminal is at location $\theta$ as $\overline{\mathbf{z}}(\boldsymbol{\theta})=\mathrm{E}[\mathbf{z} \mid \boldsymbol{\theta}]$. The difference between the measurement vector $\mathbf{z}$ and the expected value of the measurement vector for location $\boldsymbol{\theta}$ will be denoted $\tilde{\mathbf{z}}(\boldsymbol{\theta})=\mathbf{z}-\overline{\mathbf{z}}(\boldsymbol{\theta})$.

\section{A. TDoA Lower Bound}

The bounds for location will consider only the Gaussian measurement noise, not the additional scatterer measurement noise.
This does not invalidate the bounds, as the additional noise can only make the location errors increase. The conditional density of TDoA measurements given location is

$$
f_{\mathbf{Z}, \boldsymbol{\Theta}}(\mathbf{z} \mid \boldsymbol{\theta})=(2 \pi)^{-\frac{k}{2}}|\mathbf{C}|^{-\frac{1}{2}} \exp \left\{-\frac{1}{2}[\overline{\mathbf{z}}(\boldsymbol{\theta})]^{T} \mathbf{C}^{-1}[\tilde{\mathbf{z}}(\boldsymbol{\theta})]\right\} .
$$

With a little manipulation, it can be shown that

$$
\mu\left(\frac{1}{2}, \mathbf{h}\right)=\int_{S^{\prime}} \int_{\mathcal{R}^{k}} f^{\frac{1}{2}}(\mathbf{z} \mid \theta+\mathbf{h}) f^{\frac{1}{2}}(\mathbf{z} \mid \boldsymbol{\theta}) f_{\boldsymbol{\Theta}}^{\frac{1}{2}}(\boldsymbol{\theta}) f_{\mathbf{\Theta}}^{\frac{1}{2}}(\boldsymbol{\theta}+\mathbf{h}) d \mathbf{z} d \boldsymbol{\theta} .
$$

Expanding the conditional density functions gives

$$
\begin{aligned}
\mu\left(\frac{1}{2}, \mathbf{h}\right)= & \int_{S^{\prime}} \int_{\mathcal{R}^{k}}(2 \pi)^{-\frac{k}{2}}|\mathbf{C}|^{-\frac{1}{2}} \\
& \times \exp \left\{-\frac{1}{4}\left[\|\overline{\mathbf{z}}(\boldsymbol{\theta})\|_{\mathrm{C}^{-1}}^{2}\right.\right. \\
& \left.\left.\quad+\|\tilde{\mathbf{z}}(\boldsymbol{\theta}+\mathbf{h})\|_{\mathrm{C}^{-1}}^{2}\right]\right\} \\
\times & f_{\Theta}^{\frac{1}{2}}(\boldsymbol{\theta}) f_{\Theta}^{\frac{1}{2}}(\boldsymbol{\theta}+\mathbf{h}) d \mathbf{z} d \boldsymbol{\theta} .
\end{aligned}
$$

Using the standard practice when working with Gaussian probability density functions of completing squares, we obtain

$$
\begin{aligned}
\mu\left(\frac{1}{2}, \mathbf{h}\right)= & \int_{S^{\prime}} \exp \left\{-\frac{1}{2(4)}\|\overline{\mathbf{z}}(\boldsymbol{\theta})-\overline{\mathbf{z}}(\boldsymbol{\theta}+\mathbf{h})\|_{\mathbf{C}^{-1}}^{2}\right\} \\
& \times f_{\boldsymbol{\Theta}}^{\frac{1}{2}}(\boldsymbol{\theta}) f_{\boldsymbol{\Theta}}^{\frac{1}{2}}(\boldsymbol{\theta}+\mathbf{h}) \int_{\mathcal{R}^{k}}(2 \pi)^{-\frac{k}{2}}|\mathbf{C}|^{-\frac{1}{2}} \\
& \exp \left\{-\frac{1}{2}\left\|\frac{\tilde{\mathbf{z}}(\boldsymbol{\theta})+\tilde{\mathbf{z}}(\boldsymbol{\theta}+\mathbf{h})}{2}\right\|_{\mathbf{C}^{-1}}^{2}\right\} d \mathbf{z} d \boldsymbol{\theta} .
\end{aligned}
$$

By noting that the integration with respect to $\mathbf{z}$ is an integration of a probability density function and is thus unity, we obtain

$$
\begin{aligned}
\mu\left(\frac{1}{2}, \mathbf{h}\right)= & \int_{S^{\prime}} \exp \left\{-\frac{1}{2(4)}\|\overline{\mathbf{z}}(\boldsymbol{\theta})-\overline{\mathbf{z}}(\boldsymbol{\theta}+\mathbf{h})\|_{\mathbf{C}^{-1}}^{2}\right\} \\
& \times f_{\boldsymbol{\Theta}}^{\frac{1}{2}}(\boldsymbol{\theta}) f_{\boldsymbol{\Theta}}^{\frac{1}{2}}(\boldsymbol{\theta}+\mathbf{h}) d \boldsymbol{\theta}
\end{aligned}
$$

where

$$
\boldsymbol{S}^{\prime}=\boldsymbol{S} \cap\{\boldsymbol{\theta} \mid \boldsymbol{\theta}+\mathbf{h} \in \boldsymbol{S}\} .
$$

The expression in (27) is a 2-D integral no matter what the length of the measurement vector and can be computed using numerical quadrature techniques. The values for (21) can then be calculated and substituted into (17) to calculate a lower bound.

$$
\begin{aligned}
\mathbf{G}_{i j} & =\frac{\mathrm{E}\left\{\left[L^{s_{i}}\left(\mathbf{z}, \boldsymbol{\theta}+\mathbf{h}_{i}, \boldsymbol{\theta}\right)-L^{1-s_{i}}\left(\mathbf{z}, \boldsymbol{\theta}-\mathbf{h}_{i}, \boldsymbol{\theta}\right)\right]\left[L^{s_{j}}\left(\mathbf{z}, \boldsymbol{\theta}+\mathbf{h}_{j}, \boldsymbol{\theta}\right)-L^{1-s_{j}}\left(\mathbf{z}, \boldsymbol{\theta}-\mathbf{h}_{j}, \boldsymbol{\theta}\right)\right]\right\}}{\mathrm{E}\left\{L^{s_{i}}\left(\mathbf{z}, \boldsymbol{\theta}+\mathbf{h}_{i}, \boldsymbol{\theta}\right)\right\} \mathrm{E}\left\{L^{s_{j}}\left(\mathbf{z}, \boldsymbol{\theta}+\mathbf{h}_{j}, \boldsymbol{\theta}\right)\right\}} \\
\mathbf{H} & =\left[\mathbf{h}_{1}, \mathbf{h}_{2}\right]
\end{aligned}
$$




\section{B. ToA Lower Bound}

The derivation is identical to the TDoA estimator case. The $\mu(\cdot)$ function value is given by

$$
\begin{aligned}
& \mu\left(\frac{1}{2}, \mathbf{h}\right)=\int_{S^{\prime}} \exp \left\{-\frac{1}{2(4) \sigma^{2}} \sum_{j=1}^{k}\left[\mathbf{d}_{j}(\boldsymbol{\theta}+\mathbf{h})-\mathbf{d}_{j}(\boldsymbol{\theta})\right]^{2}\right\} \\
& \times f_{\Theta}^{\frac{1}{2}}(\boldsymbol{\theta}) f_{\boldsymbol{\Theta}}^{\frac{1}{2}}(\boldsymbol{\theta}+\mathbf{h}) d \boldsymbol{\theta} .
\end{aligned}
$$

Using these $\mu(\cdot)$ values, a bound can be easily calculated using (17)

\section{REFERENCES}

[1] FCC, "Report and order and further notice of proposed rulemaking in the matter of revision of the commission's rules to ensure compatibility with enhanced 911 emergency calling systems,", FCC Docket 94-102, June 1996.

[2] — "Guidelines for testing and verifying the accuracy of wireless E911 location systems,", OET Bulletin 71, Apr. 2000.

[3] Y. Zhao, "Standardization of mobile phone positioning for $3 \mathrm{G}$ systems," IEEE Commun. Mag., vol. 40, pp. 108-116, July 2002.

[4] T. Lewis, "Tracking the 'anywhere anytime' inflection point," IEEE Computer, vol. 32, pp. 134-136, Feb. 2000.

[5] Y. Zhao, "Mobile phone location determination and its impact on intelligent transportation systems," IEEE Trans. Intell. Trans. Syst., vol. 1, pp. 55-64, Mar. 2000.

[6] Z. J. Haas, J. H. Winter, and D. S. Johnson, "Simulation results on the capacity of cellular systems," IEEE Trans. Veh. Technol., vol. 46, pp. 805-817, Nov. 1997.

[7] M. D. Kulavaratharasah and A. H. Aghvami, "Teletraffic performance and evaluation of microcellular personal communication networks (PCN's) with prioritized handoff procedures," IEEE Trans. Veh. Technol., vol. 48, no. 1, pp. 137-152, 1999.

[8] A. Acampora, "Wireless ATM: a perspective on issues and prospects," IEEE Personal Commun., vol. 3, pp. 8-17, Aug. 1996.

[9] J. J. Caffery Jr and G. L. Stüber, "Subscriber location in CDMA cellular networks," IEEE Trans. Veh. Technol., vol. 47, pp. 406-416, May 1998.

[10] J. D. Parsons, The Mobile Radio Propagation Channel, 2nd ed. Toronto, ON, Canada: Wiley, 2000.

[11] Y. Qi and H. Kobayashi, "On geolocation accuracy with prior information in nonline-of-sight environment," in IEEE Fall Vehicular Technology Conf., Vancouver, BC, Canada, September 2002, pp. 285-288.

[12] M. P. Wylie and J. Holtzman, "The nonline of sight problem in mobile location estimation," in Int. Conf. Universal Personal Communications, 1996, pp. 827-831

[13] J. M. Mendel, Lessons in Estimation Theory for Signal Processing, Communications, and Control. Englewood Cliffs, NJ: Prentice-Hall, 1995.

[14] I. Jami, M. Ali, and R. F. Ormondroyd, "Comparison of methods of locating and tracking cellular mobiles," in Inst. Elect. Eng. Colloq. Novel Methods of Location and Tracking of Cellular Mobiles and Their System Applications, 1999, pp. 1/1-1/6.

[15] J. J. Caffery Jr and G. L. Stüber, "Overview of radiolocation in CDMA cellular systems," IEEE Commun. Mag., vol. 36, pp. 38-45, Apr. 1998.

[16] B. Ludden and L. Lopes, "Cellular based location technologies for UMTS: a comparison between IPDL and TA-IPDL," in IEEE Spring Vehicular Technology Conf., 2000, pp. 1348-1353.

[17] Y. T. Chan and K. C. Ho, "A simple and efficient estimator for hyperbolic location," IEEE Trans. Signal Processing, vol. 42, pp. 1905-1915, Aug. 1994.

[18] J. Winter and C. Wengerter, "High resolution estimation of the time of arrival for GSM location," in IEEE Spring Vehicular Technology Conf., 2000, pp. 1343-1347.

[19] J. H. Reed, K. J. Krizman, B. D. Woerner, and T. S. Rappaport, “An overview of the challenges and progress in meeting the E-911 requirement for location service," IEEE Commun. Mag., vol. 36, pp. 30-37, Apr. 1998.

[20] J. J. Caffery Jr, Wireless Location in CDMA Cellular Radio Systems. Boston, MA: Kluwer Academic, 2000.

[21] — "A new approach to the geometry of TOA location," in IEEE Vehicular Technology Conf., Sept. 2000, pp. 1943-1949.
[22] P.-C. Chen, "A cellular based mobile location tracking system," in IEEE Vehicular Technology Conf., May 1999, pp. 1979-1983.

[23] M. Hellebrandt and R. Mathar, "Location tracking of mobiles in cellular radio networks," IEEE Trans. Veh. Technol., vol. 48, pp. 1558-1562, Sept. 1999

[24] H.-Y. Kim, W.-S. Yoon, D. J. Kim, and Y. H. Kim, "Mobile positioning using improved least squares algorithm in cellular systems," IEICE Trans. Commun., vol. E84-B, pp. 138-140, Jan. 2001.

[25] S. M. Kay, Fundamentals of Statistical Signal Processing: Estimation Theory. Englewood Cliffs, NJ: Prentice Hall, 1993.

[26] S. Ichitsubo, T. Furono, T. Taga, and R. Kawasaki, "Multipath propagation model for line-of-sight street microcells in urban area," IEEE Trans. Veh. Technol., vol. 49, pp. 422-427, Mar. 2000.

[27] L. J. Greenstein, V. Erceg, Y. S. Yeh, and M. V. Clark, "A new path-gain/delay-spread propagation model for digital cellular channels," IEEE Trans. Veh. Technol., vol. 46, pp. 477-485, May 1997.

[28] J. B. Andersen, T. S. Rappaport, and S. Yoshida, "Propagation measurements and models for wireless communications channels," IEEE Commun. Mag., vol. 33, pp. 42-49, Jan. 1995.

[29] W. C. Y. Lee, Mobile Communications Engineering, 2nd ed. Toronto, Ontario: McGraw-Hill, 1998.

[30] D. W. Scott, Multivariate Density Estimation: Theory, Practice, and Visualization. Toronto, ON, Canada: Wiley, 1992.

[31] K. N. Plataniotis, D. Androutsos, S. Vinayagamoorthy, and A. N. Venetsanopoulos, "Color image processing using adaptive multichannel filters," IEEE Trans. Image Processing, vol. 6, pp. 933-949, July 1997.

[32] G. A. Babich and O. I. Camps, "Weighted Parzen windows for pattern classification," IEEE Trans. Pattern Anal. Machine Intell., vol. 18, pp. 567-570, May 1996.

[33] M. Barni, V. Cappellini, and A. Mecocci, "A modified metric to compare distances,” Pattern Recognit., vol. 25, no. 5, pp. 667-677, May 1992.

[34] A. Leon-Garcia, Probability and Random Processes for Electrical Engineering, 2nd ed. Don Mills, ON, Canada: Addison-Wesley, 1994.

[35] D. J. Torrieri, "Statistical theory of passive location systems," IEEE Trans. Aerosp. Electron. Syst., vol. AES-20, pp. 183-198, Mar. 1984.

[36] S. Tekinay, E. Chao, and R. Richton, "Performance benchmarking for wireless location systems," IEEE Commun. Mag., vol. 36, pp. 72-76, Apr. 1998.

[37] S. Aguirre, "Radio propagation into buildings at 912, 1920, and 5990 MHz using microcells," in Int. Conf. Universal and Personal Communications, Sept. 1994, pp. 129-134.

[38] R. D. J. Van Nee, "Spread-spectrum code and carrier synchronization errors caused by multipath and interference," IEEE Trans. Aerosp. Electron. Syst., vol. 29, pp. 1359-1365, Oct. 1993.

[39] P. Lundqvist, H. Asplund, and S. Fischer, "Evaluation of positioning systems,", Tech. Rep. T1P1.5/97-110, Dec. 1997.

[40] E. Weinstein and A. J. Weiss, "A general class of lower bounds in parameter estimation," IEEE Trans. Inform. Theory, vol. 34, pp. 338-342, Mar. 1988

[41] C. Botteron, M. Fattouche, and A. Høst-Madsen, "Statistical theory of the effects of radio location system design parameters on the positioning performance," in IEEE Vehicular Technology Conf., Vancouver, BC, Canada, Sept. 2002, pp. 1187-1191.

[42] M. A. Spirito, "On the accuracy of cellular mobile station location estimation," IEEE Trans. Veh. Technol., vol. 50, pp. 674-685, May 2001.

[43] H. L. Van Trees, Detection, Estimation, and Modulation Theory. New York: Wiley, 2001, vol. 1.

[44] R. O. Duda, P. E. Hart, and D. G. Stork, Pattern Classification, 2nd ed. Toronto, ON, Canada: Wiley, 2001.

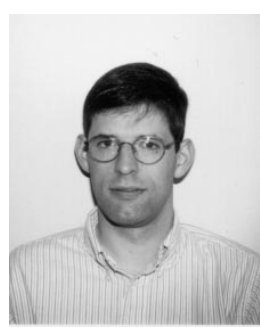

Michael McGuire (S'96-M'97) received the B.Eng. degree in computer engineering and the M.A.Sc. degree from the University of Victoria, Victoria, BC, Canada, in 1995 and 1997, respectively. He has recently received the Ph.D. degree at the Department of Electrical and Computer Engineering, University of Toronto, Toronto, ON, Canada.

His research interests are estimation and control algorithms for wireless cellular networks. 


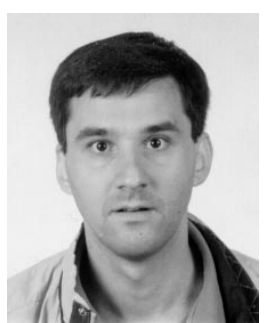

Konstantinos N. Plataniotis (S'90-M'92) received the B.Eng. degree in computer engineering from the Department of Computer Engineering and Informatics, University of Patras, Patras, Greece, in 1988 and the M.S. and Ph.D. degrees in electrical engineering from the Florida Institute of Technology, Melbourne, in 1992 and 1994, respectively.

He was a Research Associate with the Computer Technology Institute, Patras, from 1989 to 1991 . He was a Postdoctoral Fellow at the Digital Signal and Image Processing Laboratory, Department of Electrical and Computer Engineering, University of Toronto, from 1995 to 1997 From 1997 to 1999, he was an Assistant Professor with the School of Computer Science, Ryerson Polytechnic University. While there, he was a Lecturer in 12 courses for industry and continuing education programs. Since 1999, he has been with the University of Toronto as an Assistant Professor in the Department of Electrical and Computer Engineering, where he researches and teaches adaptive systems and multimedia signal processing. He is coauthor (with A. N Venetsanopoulos) of Color Image Processing and Applications (Berlin, Germany: Springer Verlag, 2000). He is a contributor to three books and has published more than 100 papers in refereed journals and conference proceedings on the areas of adaptive systems, signal and image processing, and communication systems and stochastic estimation. His current research interests include adaptive systems statistical pattern recognition, multimedia data processing, statistical communication systems, and stochastic estimation and control. He was Technical Cochair of the Canadian Conference on Electrical and Computer Engineering (CCECE 2001, May 13-16, 2001, Toronto).

Dr. Plataniotis is a member of the IEEE Technical Committee on Neural Networks for Signal Processing.

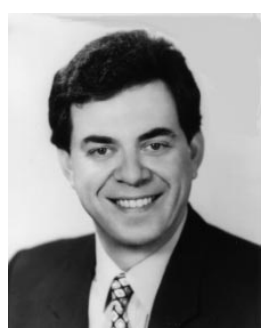

Anastasios N. Venetsanopoulos (S'66-M'69SM'79-F' 88 ) received the diploma in engineering degree from the National Technical University of Athens (NTU), Greece, in 1965 and the M.S., M.Phil., and Ph.D. degrees in electrical engineering from Yale University, New Haven, CT, in 1966, 1968, and 1969, respectively.

He joined the Department of Electrical and Computer Engineering, University of Toronto, Toronto, ON, Canada, in September 1968 as a Lecturer and became Assistant Professor in 1970, Associate Professor in 1973, and Professor in 1981. Since July 1997, he has been Associate Chair: Graduate Studies of the Department of Electrical and Computer Engineering. He was Acting Chair during the spring term of 1998-1999. In July 1999, he became Inaugural Chairholder in Multimedia. He is Dean of the Faculty of Applied Science and Engineering, University of Toronto. He has served as Chair of the Communications Group and Associate Chair of the Department of Electrical Engineering. He was on research leave at Imperial College of Science and Technology, NTU, the Swiss Federal Institute of Technology, the University of Florence, and the Federal University of Rio de Janeiro. He was an Adjunct Professor at Concordia University. He has been a Lecturer in 138 short courses for industry and continuing education programs and a Consultant to numerous organizations. He is a contributor to 29 books and coauthor of Nonlinear Digital Filters: Principles and Applications, Boston, MA: Kluwer, 1990 Artificial Neural Networks: Learning Algorithms, Performance Evaluation and Applications, Boston, MA: Kluwer, 1993; Fuzzy Reasoning in Information, Decision, and Control Systems, Boston, MA: Kluwer, 1994; and Color Image Processing and Applications, Berlin, Germany: Springer-Verlag, 2000. He has published more than 680 papers in refereed journals and conference proceedings on digital signal and image processing and digital communications. He was President of the Canadian Society for Electrical Engineering and Vice President of the Engineering Institute of Canada (EIC) (1983-1986). He was Editor of the Canadian Electrical Engineering Journal (1981-1983).

Prof. Venetsanopoulos is a Fellow of the EIC and the Canadian Academy of Engineering. He is a member of Sigma Xi, the Technical Chamber of Greece, the European Association of Signal Processing, and the Association of Professional Engineers of Ontario (APEO) and Greece. He has served as Chair on numerous boards, councils, and technical conference committees of the IEEE, such as the Toronto Section (1977-1979) and the IEEE Central Canada Council (1980-1982). He was Guest Editor or Associate Editor for several IEEE journals. He is a member of the IEEE Communications, Circuits and Systems, Computer, and Signal Processing Societies. He received an Honorary Doctorate from NTU in October 1994. In October 1996, he received the Excellence in Innovation Award from the Information Technology Research Centre of Ontario and Royal Bank of Canada for "innovative work in color image processing and its industrial applications." In November 2000, he received the Millennium Medal from the IEEE. 